\title{
The National Autism Database of Israel: a Resource for Studying Autism Risk Factors, Biomarkers, Outcome Measures, and Treatment Efficacy
}

\author{
Ilan Dinstein ${ }^{1,2,3}$ - Ayelet Arazi ${ }^{2,3} \cdot$ Hava M. Golan ${ }^{3,4} \cdot$ Judah Koller $^{5}$ • Evan Elliott ${ }^{6}$ - Illana Gozes ${ }^{7}$. Cory Shulman ${ }^{8}$. \\ Sagiv Shifman ${ }^{9} \cdot$ Raanan Raz $^{10} \cdot$ Nadav Davidovitch $^{11} \cdot$ Tali Gev $^{12,13}$. Adi Aran ${ }^{14,15} \cdot$ Orit Stolar $^{16}$.

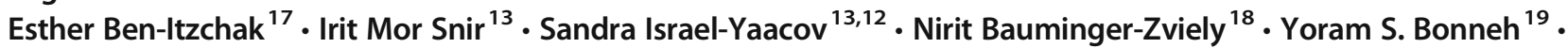 \\ Eynat Gal ${ }^{20}$ - Simone Shamay-Tsoory ${ }^{21}$ - Anat Zaidman Zait ${ }^{22,23}$ - Bat Sheva Hadad ${ }^{24,25} \cdot$ Raz Gross $^{26,27}$. \\ Michal Faroy ${ }^{3,28}$. Eitan Bachmat ${ }^{29}$. Alal Eran ${ }^{30}$. Florina Uzefovsky ${ }^{1}$ - Hagit Flusser ${ }^{3,31}$ - Analya Michaelovski ${ }^{3,31}$. \\ Stephen Z. Levine ${ }^{32}$. Arad Kodesh ${ }^{32,33}$. Doron Gothelf ${ }^{26,34,35}$. Daphna Marom ${ }^{26,36}$ - Hagit Baris Feldman ${ }^{26,36}$. \\ Dalit Ben Yosef $^{37,38}$. Aviva Mimouni Bloch ${ }^{26,39}$. Yair Sadaka ${ }^{40}$. Chen Schtaierman ${ }^{41}$ - Michael Davidovitch ${ }^{42}$. \\ Michal Begin ${ }^{43} \cdot$ Lidia V. Gabis ${ }^{26,35,44} \cdot$ Ditza Zachor ${ }^{16,26} \cdot$ Idan Menashe ${ }^{3,45} \cdot$ Ofer Golan $^{13,12,46} \cdot$ Gal Meiri $^{3,28}$
}

Published online: 28 July 2020

(C) Springer Science+Business Media, LLC, part of Springer Nature 2020

\section{Introduction}

ASD is a neurodevelopmental disorder that is defined by impairments in social communication, restricted interests, and repetitive behaviors (American Psychiatric Association 2013). Despite the unitary definition, individuals with ASD exhibit a wide variety of different core and secondary symptoms, which include dramatic differences in the level of adaptive behaviors, language, and cognitive abilities. This heterogeneity suggests that the ASD diagnostic category includes a variety of distinct disorders (Happé et al. 2006) that develop due to different causes (State and Levitt 2011; Jeste and Geschwind 2014) and are likely to require different interventions and therapies (Zwaigenbaum et al. 2015).

Consequently, conducting ASD research with small groups of participants in isolated laboratories yields findings that are not likely to be replicated across sites. The alternative, which has gained considerable momentum over the last decade, is to develop collaborative research efforts that involve identical data

Ilan Dinstein and Ayelet Arazi contributed equally to this work.

This study was funded by the Israeli Ministry of Science and Technology through a National Knowledge Center grant awarded to I.D, I.M, H.G, and G.M.

Ilan Dinstein

dinshi@bgu.ac.il

Illana Gozes

igozes@tauex.tau.ac.il

Extended author information available on the last page of the article collection at multiple sites and the establishment of a common shared database. Such efforts enable data collection from a larger number of participants who are more likely to represent the true heterogeneity of ASD characteristics in the community. This approach can, therefore, enable researchers to pursue a personalized medicine approach with the goal of dividing the heterogeneous population into distinct subgroups that share specific phenotypic features, etiologies and/or treatment response patterns. These efforts are considerably more difficult and expensive to establish, and require multidisciplinary collaboration.

Several successful examples of such collaborations include the Simons Simplex Collection (Fischbach and Lord 2010) and SPARK (Feliciano et al. 2018) databases that focus on revealing the genetics of ASD. The EU-AIMS Longitudinal European Autism Project (Loth et al. 2017), which aims to identify distinct biomarkers for ASD using neuroimaging, behavioral assessments, and biological samples from hundreds of individuals with ASD who are sampled longitudinally over several years. The Autism Brain Imaging Data Exchange (ABIDE) project (Di Martino et al. 2014), which has aggregated hundreds of brain MRI scans from 6 to 60 years old individuals with ASD along with Autism Diagnostic Observation Schedule (ADOS) and cognitive assessment scores. Finally, until recently, the Autism Treatment Network (ATN), which included a consortium of sites in North America that collected a wide variety of behavioral and clinical data from thousands of ASD cases with the goal of improving clinical guidelines for best practice (Coury et al. 2020).

Recently, several countries have begun to create national autism databases in an effort to develop ASD research that is focused on local communities. Given the vast heterogeneity of 
ASD, one may assume that different ethnic communities and geographic locations with different genetics, environments, education systems, health resources, and cultural norms will require different solutions. Examples of such work include the Australian Autism Biobank (Alvares et al. 2018) and the Italian Autism Network (Muglia et al. 2018).

In 2018, the Israeli Ministry of Science and Technology awarded a grant to scientists at Ben Gurion University (BGU) and physicians at the neighboring Soroka University Medical Center (SUMC) to create the National Autism Research Center of Israel (NARCI). The first goal of the center was to turn a previously regional autism database (Meiri et al. 2017) into a national database with standardized data collection at multiple clinical sites throughout the country. This supports the center's vision to improve autism diagnosis and treatment in Israel by integrating multidisciplinary research into the social healthcare system of Israel. In February 2019, we conducted the first Israeli Meeting for Autism Research where leading Israeli autism researchers and clinicians discussed shared research goals and defined the current study protocol for the NARCI database.

\section{Methods}

\section{Study Design}

This national project takes advantage of the centralized structure of the social healthcare system in Israel, which is run by four Health Maintenance Organizations (HMOs) that are obligated to accept any Israeli citizen and provide all citizens with equal medical services that are either free of charge or at very low cost. Within this framework, children who are suspected of having ASD are referred to one of $\sim 50$ clinics, mostly located in child development centers (with $\sim 10$ of these clinics handling $\sim 70 \%$ of national referrals). Approximately 1800 new cases of ASD are diagnosed annually in these clinics, and in many of them, children are invited to periodic follow-ups that are free of charge. Diagnostic protocols vary across sites, with some clinics performing standardized ADOS and cognitive and language assessments while others perform partial assessments or more limited clinical evaluations. The formal ASD diagnosis in Israel must be performed according to DSM-5 criteria and include separate diagnoses from both a psychologist and a physician (pediatric neurologist or child psychiatrist).

Thus far, the NARCI has developed partnerships with four such clinics: The Preschool Psychiatry Unit at SUMC, the Child Development Center at SUMC, the Health Ministry Child Development Center at Beer Sheva, and the ALUT Autism Center at Shamir Medical Center. When necessary, the NARCI provides the clinical teams with the necessary knowledge, training, and additional work force to create standardized and reliable assessment protocols across sites. This is a cost-effective way to create clinical research partnerships that simultaneously enable autism research and improve clinical care. It is worth noting that this framework is ideal for conducting multi-site clinical trials and longitudinal research regarding treatment efficacy. Identical research protocols have received ethical approval at the four participating sites where $\sim 450$ new cases of ASD are diagnosed annually. Additional sites will be added in the near future.

Critical components of the study design include standardized data collection at the time of diagnosis and at follow-up visits, subsequent incorporation of the data into identical database structures, and the eventual integration of anonymized data across sites. Database administration is handled centrally by the NARCI with research assistants managing data collection and processing at each of the sites to ensure consistency and reliability.

\section{Selected Scientific Questions}

Over 40 leading Israeli autism researchers and clinicians participated in identifying the following nine shared scientific questions that were agreed to be the highest priority for ASD research in Israel. The database is designed to address these key scientific questions:

1. Are there distinct clinical profiles (i.e., subgroups) in the ASD population that can be defined based on specific behavioral, genetic, and/or other biological measures?

2. How effective are current diagnostic protocols and screening methods in Israel, and how can they be improved?

3. Which techniques and technologies enable objective, reliable, and sensitive early detection of ASD and quantification of ASD symptoms (e.g., outcome measures)?

4. How effective are existing community-based treatments and educational settings in reducing core and secondary ASD symptoms. How can their efficacy be improved?

5. How effective are new behavioral, pharmacological, and other medical interventions in reducing core and secondary ASD symptoms?

6. Which behavioral and/or biological measures enable accurate prognosis of short- and long-term outcomes for individuals with ASD?

7. What are the genetic and environmental risk factors for ASD in Israel?

8. How prevalent are various medical comorbidities in Israeli children with ASD?

9. What are the factors that influence the functioning and well-being of families of children with ASD in Israel?

After selecting the scientific questions, we developed a list of the most important behavioral and biological measures that are necessary for addressing these questions. In consideration of practical limitations, we selected measures that would be 
feasible given limitations in budget, personnel, and availability of participants.

\section{Participants}

Children, between the ages of 1 and 8 years old, who were referred with a suspicion of ASD to one of the participating clinics, are eligible to participate if they have completed the ADOS assessment. To facilitate recruitment, it is enough that one parent signs the informed consent form on behalf of both parents. Either parent can stop participation in the research at any time, and the family will be excluded from further research. To date, 961 children and their parents have been recruited at the four sites. This cohort exhibits the typical 4:1 male to female ratio and a wide distribution of ADOS and cognitive scores demonstrating heterogeneous severity levels (Fig. 1). Initial analyses demonstrate moderately negative correlations between ADOS-calibrated severity scores and cognitive scores $(r=-0.38, p<0.0001)$ suggesting that children with more severe ASD symptoms tend to have lower cognitive abilities. Age of diagnosis was negatively correlated with the ADOS-calibrated severity scores $(r=-0.27, p<0.0001)$ and positively correlated with cognitive scores $(r=0.17$, $p<0.0001)$ suggesting that children who are diagnosed earlier tend to have more severe ASD symptoms and poorer cognitive abilities. Maternal and paternal age at birth were not significantly correlated with symptom severity $(-0.1<r<0.1$, $p>0.1$.

Since the majority of the children currently in the database are from the south of Israel (i.e., diagnosed at SUMC), 22\% of the children are from the Bedouin nomadic Arab community. This is a unique tribal community with $\sim 66 \%$ consanguineous marriages (i.e., first cousins) and frequent polygamy with very large families, which offers a singular context for genetic studies of ASD. In addition to the mandatory ADOS assessments, a variety of other data has been collected from the children and their families (Table 1). The types and sources of the data are described below.

\section{Behavioral Assessments}

A fundamental necessity of all ASD studies is to acquire standardized behavioral assessments that capture basic phenotypic
Fig. 1 Characteristics of the children who are currently in the database $(N=961)$. a Gender and ethnicity. b Age at diagnosis. c Cognitive scores at diagnosis. $\mathbf{d}$ ADOS-calibrated severity scores (i.e., comparison score) at diagnosis. e Maternal age at birth. f Paternal age at birth a

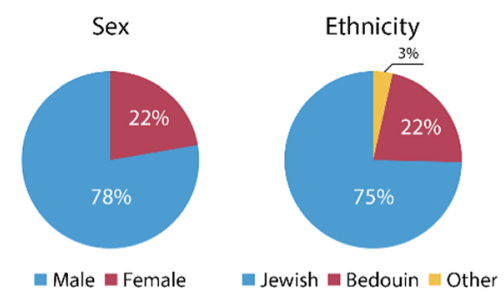

C

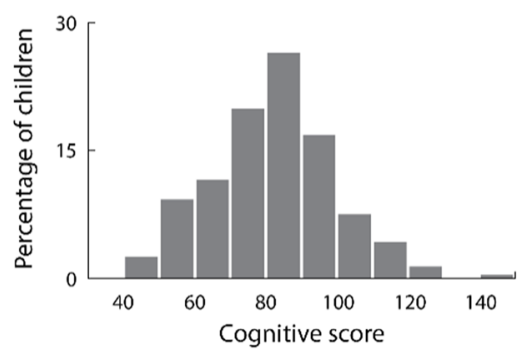

e

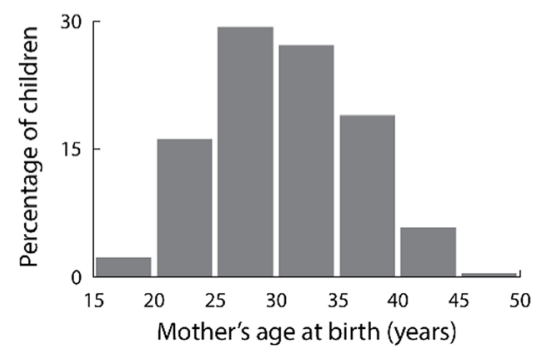

b

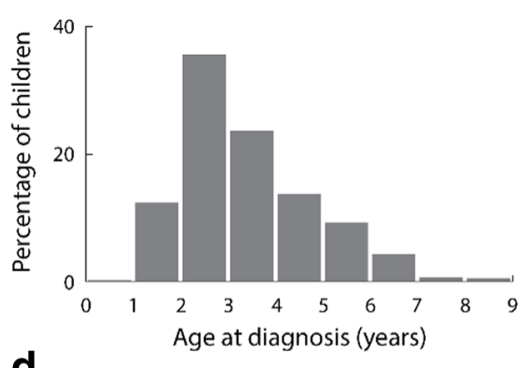

d

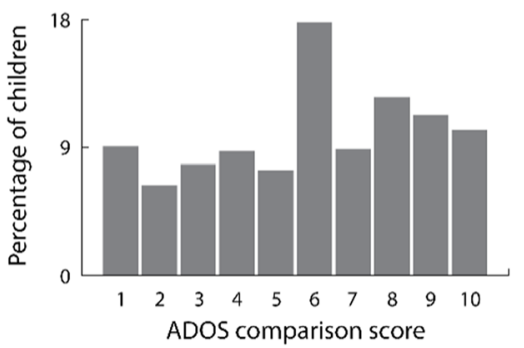

f

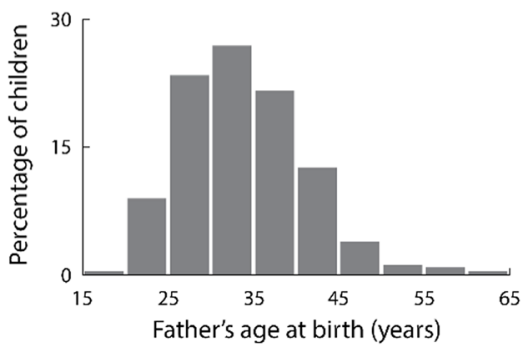


Table 1 Current availability of different types of information from children who were recruited to the database

\begin{tabular}{lc}
\hline Type of data & $N$ children (\%) \\
\hline Behavioral assessments & $961(100)$ \\
ADOS-2 & $558(58)$ \\
DSM-5 & $295(31)$ \\
PLS-3 & $693(72)$ \\
Cognitive test (one of the following) & $446(46)$ \\
1. Bayley & $35(4)$ \\
2. Mullen & $212(22)$ \\
3. WPPSI & \\
Interviews and questionnaires & $794(83)$ \\
Intake questionnaire & $359(38)$ \\
Sensory Profile 2 & $537(56)$ \\
CSHQ & $74(8)$ \\
ABC & $341(35)$ \\
Adaptive behaviors (one of the following) & $27(3)$ \\
1. Vineland-2 & $314(33)$ \\
2. ABAS-2 & $732(76)$ \\
Audio and video recordings & $319(33)$ \\
Birth and medical records extracted so far (records are available for $~ 80 \%$ of the children) \\
MRI scans extracted so far (scans are available for $~ 20 \%$ of the children) & $63(7)$ \\
DNA samples (whole exome sequencing) & $170(18)$ \\
\hline
\end{tabular}

features from children at multiple ages. The following assessments were selected:

- DSM-5 assessment (American Psychiatric Association 2013): Physicians and psychologists assess the DSM-5 criteria for ASD in a meeting with the child and parents. The results of this assessment are recorded in a standardized form including the level of required support in each symptom domain.

- Autism Diagnostic Observation Schedule 2 (ADOS-2) (Lord et al. 2012): Trained clinicians administer this standardized ASD assessment of the child. The ADOS yields scores describing the severity of symptoms in the social and repetitive behavior domains and is a requirement for entry into the database.

- Preschool Language Scale 3 (PLS-3) (Volden et al. 2011): Speech pathologists administer this standardized language assessment, which yields scores regarding speech production and comprehension.

- One of the following cognitive assessments, administered by a licensed psychologist:

1. Bayley Scales of Infant and Toddler Development (Viezel et al. 2014): cognitive test for children < 3.5 years old that yields a developmental quotient score with a distribution that is equivalent to IQ scores (population mean of 100 and a standard deviation of 15).
2. Mullen Scales of Early Learning (Mullen 1995): cognitive test for children $<5$ years old that yields a composite score of early learning abilities with an equivalent distribution to IQ scores.

3. Wechsler Preschool and Primary Scale of Intelligence (WPPSI) (Luiselli et al. 2013): standardized IQ test for children 2.5-7.7 years old.

\section{Audio and Video Recordings}

Recent advancements in computer vision and speech analysis suggest that certain ASD symptoms may be identified and quantified by analyzing video and audio recordings of children with ASD (Budman et al. 2019; Sadiq et al. 2019). To enable such research, a subset of the ADOS and cognitive and language assessments in all four clinical sites are performed in rooms equipped with audio and video recording systems. When recorded, parents sign an additional consent form approving the use of recordings for clinical and research purposes.

\section{Interviews and Questionnaires}

In addition to behavioral assessments, basic phenotypic information is gathered using the following parent interviews and questionnaires: 
- Intake questionnaire with the following information:

1. Sociodemographic-education, income, ethnicity, age, history of addresses

2. Family history-number of children, miscarriages, medical history in the immediate and extended family, medications, smoking, alcohol, substance abuse

3. Pregnancy - use of assisted reproductive technologies, vitamins and supplements, smoking, alcohol, medications, high-risk pregnancy, illnesses, complications

4. Birth-gestational age, weight, complications, neonatal care unit

5. Early development-medical history, medications, vaccinations, feeding difficulties, physical growth, motor development, language development, regression

6. Early interventions-history of early interventions and educational placements.

- Assessment of adaptive behaviors using one of the following options:

1. Vineland Adaptive Behavior Scales 2nd Ed. (Sparrow et al. 2005): a structured parent interview.

2. Adaptive Behavior Assessment System 2 (ABAS-2)

(Harrison and Oakland 2003): parent questionnaire.

- Sensory Profile 2 (Dunn and Westman 1996): This is a parent questionnaire for evaluating hypo- and hyper- sensitivities in a variety of sensory modalities.

- The Children's Sleep Habits Questionnaire (CSHQ) (Owens et al. 2000): This is a parent questionnaire for evaluating the severity of sleep disturbances in children.

- Aberrant Behaviors Checklist (ABC) (Aman et al. 1985): This is a parent questionnaire for evaluating the severity of aberrant behaviors in children.

Additional questionnaires, necessary for addressing key scientific questions, will be added in the near future. These include measures of parental characteristics such as the Parenting Stress Index (Abidin et al. 2013) and Beck Depression Inventory (Jackson-Koku 2016), measures of children's ADHD symptoms such as the Conner's rating scale (Keith Conners et al. 1998), measures of children's anxiety symptoms such as the SCARED questionnaire (Birmaher et al. 1999), and ASD screening measures such as the Social Responsiveness Scale (Constantino 2012), and the Modified Checklist for Autism in Toddlers (Robins et al. 2001).

\section{Birth and Medical Records}

The approved parental consent form authorizes the research team to extract information from the birth and medical record of the child, which are available for most of the children through their Health Maintenance Organization (HMO) electronic records. This includes the following information:

1. Birth - gestational age, weight, Apgar score, complications, newborn hearing test, neonatal intensive care unit

2. Service use-hospitalizations, medications, referrals, blood tests, and medical exams

3. Comorbidities - current and previous illnesses and disorders

4. Development - physical growth, motor and language milestones, and vaccinations

\section{MRI Scans}

Surprisingly, initial examination of the available medical records revealed that $\sim 20 \%$ of the children in the database were referred to a clinical brain MRI at some point before or after their ASD diagnosis. Clinical brain MRI scans in Israel typically include a detailed anatomical $1 \times 1 \times 1$-mm T1-weighted scan and a lower resolution $2 \times 2 \times 2$-mm diffusion-weighted scan (at least 6 directions). The parental consent form allows us to extract the scans from the HMO database and perform additional analyses with the raw data.

\section{Genetic Evaluations}

Saliva samples are collected from participating children and both parents for extraction of DNA. The DNA is sent to the Autism Sequencing Consortium (Satterstrom et al. 2020) for whole exome sequencing. The resulting sequences enable studies that assess the prevalence of de novo and inherited genetic abnormalities in the Israeli population. Furthermore, it is possible to relate specific genetic abnormalities to the deep phenotypic data available for this cohort. Participation in the genetic study requires both parents to sign an additional consent form that has been approved at all four sites.

\section{Biological Samples}

Protocols for collecting, processing, and storing additional biological samples including blood, stool, urine, and hair samples have already been approved through participation in the Israeli Biorepository Network for Research (MIDGAM, www.midgam.org.il). The MIDGAM is a national organization operating in five major medical centers in Israel with infrastructure for processing and storing a variety of biological samples. Participation will require parents to sign an additional consent form. Collection of biological samples is expected to begin in late 2020 . 


\section{Longitudinal Data Collection}

Within ASD research, it is essential to study how behavioral and biological measures change over time, particularly in the context of intervention (Georgiades et al. 2017). This is critical for revealing the different etiologies of autism, for developing prognostic measures, and for assessing treatment efficacy. With this in mind, a strong emphasis of the NARCI database is to enable longitudinal data collection at participating sites using identical standardized measures as in the initial diagnosis. While most ASD clinics in Israel invite families for follow-up visits 1-2 years after the initial diagnosis, these visits are typically short and do not include the same behavioral assessments performed at diagnosis.

To counteract this challenge, the NARCI is funding the administration of follow-up behavioral assessments at each of the clinical sites. Data collection at follow-up currently includes questionnaires regarding adaptive behaviors, aberrant behaviors, sensory sensitivity, sleep disturbances, and a follow-up questionnaire regarding the child's educational placement, behavioral interventions, and use of medications. In addition, standardized ADOS, cognitive, and language assessments are performed by NARCI personnel (Table 2). Follow-up assessments have been completed with 208 children at the two SUMC sites, with expansion to the other sites planned this year (Table 2).

\section{Data Management and Sharing}

To ensure privacy, identifiable data is held in separate local password-protected databases at each of the clinical sites (with the exception of the two SUMC sites where data management is combined). The databases reside on their respective medical center's internal computer networks, which are protected according to the standards enforced by each center. Research assistants from the NARCI join the local clinical teams at each

Table 2 Current availability of different types of information for children who completed a follow-up assessment 1-2 years after the initial diagnosis

\begin{tabular}{lc}
\hline Type of data & $N$ children $(\%)$ \\
\hline Behavioral assessments & \\
ADOS-2 & $208(100)$ \\
PLS-3 & $34(16)$ \\
Cognitive assessment & $133(64)$ \\
Questionnaires & \\
Follow-up questionnaire & $208(100)$ \\
ABAS-2 & $32(15)$ \\
Sensory Profile 2 & $110(53)$ \\
CSHQ & $126(61)$ \\
\hline
\end{tabular}

of the sites and receive authorization from each medical center to access the clinic's computer network. They then populate identical databases that are built in access (Microsoft Inc., USA). An automated procedure de-identifies the data and enables integration of the data across sites. All clinicians and researchers associated with the participating sites have access to the anonymized, integrated data, which can be extracted for analyses in their respective laboratories. Plans are underway to enable a method for sharing the anonymized data with researchers outside of the participating sites, given appropriate ethics approval. Some forms of data, such as video recordings, cannot be anonymized and are therefore analyzed within the confines of each clinical site.

\section{Discussion}

The NARCI database is a large shared resource for performing multidisciplinary research regarding ASD risk factors, biomarkers, outcome measures, and treatment efficacy. The study protocol focuses on ASD development and incorporates retrospective data from pregnancy, birth, and medical records along with prospective data that is collected as part of the diagnosis process and in follow-up visits. The wealth of longitudinal behavioral, clinical, and biological data from this large dataset is critical for studying the heterogeneity of ASD etiologies and specifically for understanding ASD development, risk factors, diagnosis, treatment efficacy, and service utilization in the Israeli population.

\section{Research Focus}

A central goal of the NARCI is to facilitate clinical research that will improve ASD diagnosis and treatment in Israel. Such clinical research is carried out by many researchers in different fields within social sciences, medicine, engineering, and life sciences who use distinct techniques and measures. Despite differences across fields, the vast majority of clinical studies require thorough behavioral characterization of participants using standardized tests of autism severity (e.g., ADOS), cognitive function, language abilities, and adaptive behaviors. Additional measures of aberrant behaviors, sleep problems, and sensory sensitivities add critical information as do medical records containing medication use and diagnosed comorbidities. Collecting these behavioral data from a large number of 1-8-year-old children at the time of ASD diagnosis is the primary focus of the database. This is demonstrated by the large number of children for whom these data are already available (Table 1).

After establishing standardized behavioral assessments at each of the sites, we added video and audio capture of behavioral assessments, collection of saliva samples for whole exome sequencing, extraction of retrospective clinical records 
including pregnancy and birth records, collection of eye tracking data, and clinical MRI scans. Collecting these medical and biological data from a large number of 1-8-year-old children at the time of ASD diagnosis is the secondary focus of the database. We plan to add several types of data to this secondary focus including the collection of additional biological samples (blood, hair, stool, and urine) for processing and storage at MIDGAM, and collection of additional questionnaires including parent and family characteristics/function questionnaires.

After establishing the collection of these data during the ASD diagnosis, we developed a routine for collecting longitudinal information at the annual follow-up visits that families complete in most of the ASD clinics. Collection of longitudinal data follows the same priorities as initial data collection with a primary focus on standardized behavioral assessments and a secondary focus on additional medical and biological data (Table 2).

\section{Size Versus Depth}

When deciding on the research goals of the NARCI database, we were forced to choose between collecting restricted information on a relatively large number of participants (e.g., by mining the HMO records of all ASD individuals in Israel) or collecting in-depth information from a relatively small number of participants. The main reason for choosing the latter stems from the understanding that ASD heterogeneity requires in-depth characterization of participants using standardized clinical measures that involve direct interaction with the child. Standardization of measures such that they are collected in a reliable manner across sites is paramount for the success of this research. Deeper data collection limits the number of participants, thereby reducing the size of the database.

Note that this trade-off between size and depth is apparent in other collaborative research efforts around the globe. For example, genetic projects with larger cohorts (e.g., the SPARK project with a target cohort of 50,000 ASD individuals) typically reduce the depth of data collected from each participant (e.g., SPARK collects phenotypic data using parent and self-report questionnaires). Conversely, projects that collect more detailed longitudinal phenotypic and/or neuroimaging data tend to recruit smaller cohorts (e.g., the EU-AIMS LEAP project with $\sim 400$ participants).

\section{Limitations of Large-Scale Research}

While large multi-site collaborations are essential for studying large numbers of ASD individuals and understanding the heterogeneity of ASD, they also have several limitations. First, large multi-site collaborations are difficult to manage since participating members sometimes want to pursue different goals. Second, large collaborations tend to respond more slowly to emerging challenges and opportunities. Third, large collaborations are always limited in their scope such that some scientific topics and fields are left out.

Our hope is that these limitations will not impede our efforts to identify distinct subgroups of ASD children with specific behavioral and/or biological characteristics who benefit more from specific interventions and treatments. This step will enable more focused research with specific subgroups in highly specialized labs. We believe that ASD research will profit greatly from a balance between large-scale collaborative research and focused highly specialized research.

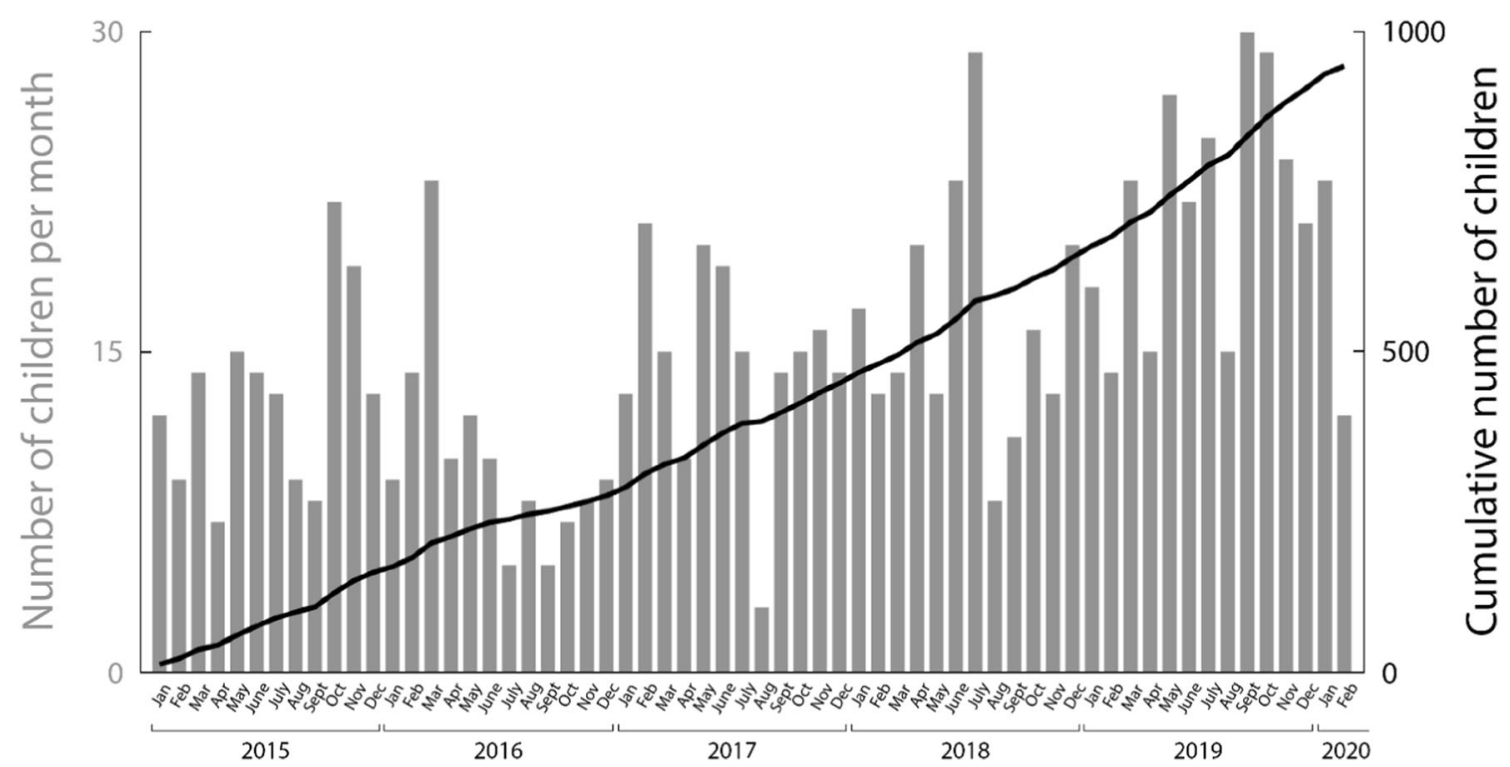

Fig. 2 Recruitment rate of new families to the database. Gray bars represent the number of children recruited per month, from January 2015 to February 2020. Black line indicates the cumulative recruitment rate. Average recruitment rate is 14.7 families per month and $\sim 180$ families per year $(N=961)$ 


\section{Recruitment Progress to Date}

Recruitment of families to the database has progressed at an average rate of $\sim 180$ new families per year (Fig. 2). The experience with the families has been extremely positive, with $\sim 80 \%$ of referred families agreeing to participate in the database. We believe that this high recruitment rate is due to the integration of the research into the ASD clinics. This enables families to participate without requiring any additional effort (i.e., research is carried out as part of the routine clinical visits). Expansion of the research to additional sites throughout Israel is planned to increase recruitment rates and data collection over the next several years. Our goal is to reach $70 \%$ of the 1800 annually diagnosed children in Israel.

\section{Conclusions and Future Plans}

The NARCI database is a unique and valuable resource for studying the development of ASD. We believe that further development of the database will enable national studies, which will reveal important findings that are unique to Israel, as well as international studies that will reveal commonalities between the Israeli and the global ASD population. Future plans also include extending the age range of participants to include adolescents and adults with ASD who require immediate solutions for a variety of challenges.

\section{References}

Abidin R, Flens JR, Austin WG (2013) The parenting stress index. In: Forensic uses of clinical assessment instruments

Alvares GA, Dawson PA, Dissanayake C et al (2018) Study protocol for the Australian autism biobank: an international resource to advance autism discovery research. BMC Pediatr 18:284. https://doi.org/10. 1186/s12887-018-1255-Z

Aman MG, Singh NN, Stewart AW, Field CJ (1985) The aberrant behavior checklist: a behavior rating scale for the assessment of treatment effects. Am J Ment Defic

American Psychiatric Association (2013) Diagnostic and statistical manual of mental disorders, 5th edn. American Psychiatric Publishing, Arlington, VA

Birmaher B, Brent DA, Chiappetta L et al (1999) Psychometric properties of the screen for child anxiety related emotional disorders (SCARED): a replication study. J Am Acad Child Adolesc Psychiatry 38:1230-1236. https://doi.org/10.1097/00004583199910000-00011

Budman I, Meiri G, Ilan M, Faroy M, Langer A, Reboh D, Michaelovski A, Flusser H, Menashe I, Donchin O, Dinstein I (2019) Quantifying the social symptoms of autism using motion capture. Sci Rep 9: 7712. https://doi.org/10.1038/s41598-019-44180-9

Constantino J (2012) Social responsiveness scale (SRS-2). West Psychol Serv

Coury DL, Murray DS, Fedele A, Hess T, Kelly A, Kuhlthau KA (2020) The autism treatment network: bringing best practices to all children with autism. Pediatrics 145:S13-S19. https://doi.org/10.1542/2019$1895 \mathrm{D}$

Di Martino A, Yan CG, Li Q et al (2014) The autism brain imaging data exchange: towards a large-scale evaluation of the intrinsic brain architecture in autism. Mol Psychiatry 19:659-667. https://doi.org/ $10.1038 / \mathrm{mp} .2013 .78$

Dunn W, Westman K (1996) The Sensory Profile: national sample of. Am J Occup Ther 50:603-604

Feliciano P, Daniels AM, Green Snyder LA, Beaumont A, Camba A, Esler A, Gulsrud AG, Mason A, Gutierrez A, Nicholson A, Paolicelli AM, McKenzie AP, Rachubinski AL, Stephens AN, Simon AR, Stedman A, Shocklee AD, Swanson A, Finucane B, Hilscher BA, Hauf B, O'Roak BJ, McKenna B, Robertson BE, Rodriguez B, Vernoia BM, van Metre B, Bradley C, Cohen C, Erickson CA, Harkins C, Hayes C, Lord C, Martin CL, Ortiz C, Ochoa-Lubinoff C, Peura C, Rice CE, Rosenberg CR, Smith CJ, Thomas C, Taylor CM, White LC, Walston CH, Amaral DG, Coury DL, Sarver DE, Istephanous D, Li D, Nugyen DC, Fox EA, Butter EM, Berry-Kravis E, Courchesne E, Fombonne EJ, Hofammann E, Lamarche E, Wodka EL, Matthews ET, O'Connor E, Palen E, Miller F, Dichter GS, Marzano G, Stein G, Hutter H, Kaplan HE, Li H, Lechniak H, Schneider HL, Zaydens H, Arriaga I, Gerdts JA, Cubells JF, Cordova JM, Gunderson J, Lillard J, Manoharan J, McCracken JT, Michaelson JJ, Neely J, Orobio J, Pandey J, Piven J, Scherr J, Sutcliffe JS, Tjernagel J, Wallace J, Callahan K, Dent K, Schweers KA, Hamer KE, Law JK, Lowe K, O’Brien K, Smith K, Pawlowski KG, Pierce KL, Roeder K, Abbeduto LJ, Berry LN, Cartner LA, Coppola LA, Carpenter L, Cordeiro L, DeMarco L, Grosvenor LP, Higgins L, Huang-Storms LY, Hosmer-Quint L, Herbert LM, Kasparson L, Prock LM, Pacheco LD, Raymond L, Simon L, Soorya LV, Wasserburg L, Lazar M, Alessandri M, Brown M, Currin MH, Gwynette MLF, Heyman M, Hale MN, Jones M, Jordy M, Morrier MJ, Sahin M, Siegel MS, Verdi M, Parlade MV, Yinger M, Bardett N, Hanna N, Harris N, Pottschmidt N, Russo-Ponsaran N, Takahashi N, Ousley OY, Juarez AP, Manning P, Annett RD, Bernier RA, Clark RD, Landa RJ, Goin-Kochel RP, Remington R, Schultz RT, Brewster SJ, Booker S, Carpenter S, Eldred S, Francis S, Friedman SL, Horner S, Hepburn S, Jacob S, Kanne S, Lee SJ, Mastel SA, Plate S, Qiu S, Sandhu S, Thompson S, White S, Myers VJ, Singh V, Yang WS, Warren Z, Amatya A, Ace AJ, Chatha AS, Lash AE, Negron B, Rigby C, Ridenour C, Stock CM, Schmidt D, Fisk I, Acampado J, Nestle JL, Nestle JA, Layman K, Butler ME, Kent M, Mallardi MD, Carriero N, Lawson N, Volfovsky N, Edgar R, Marini R, Rana R, Ganesan S, Shah S, Ramsey T, Chin W, Jensen W, Krentz AD, Gruber AJ, Sabo A, Salomatov A, Eng C, Muzny D, Astrovskaya I, Gibbs RA, Han X, Shen Y, Reichardt LF, Chung WK (2018) SPARK: a US cohort of 50,000 families to accelerate autism research. Neuron 97:488-493. https://doi.org/10.1016/j. neuron.2018.01.015

Fischbach GD, Lord C (2010) The Simons Simplex Collection: a resource for identification of autism genetic risk factors. Neuron 68: 192-195

Georgiades S, Bishop SL, Frazier T (2017) Editorial perspective: longitudinal research in autism-introducing the concept of 'chronogeneity'. J Child Psychol Psychiatry Allied Discip

Happé F, Ronald A, Plomin R (2006) Time to give up on a single explanation for autism. Nat Neurosci 9:1218-1220. https://doi.org/10. 1038/nn1770

Harrison P, Oakland T (2003) Adaptive Behavior Assessment System (ABAS-II)

Jackson-Koku G (2016) Beck depression inventory. Occup Med (Chic Ill) $66: 174-175$

Jeste SS, Geschwind DH (2014) Disentangling the heterogeneity of autism spectrum disorder through genetic findings. Nat Rev Neurol 10: 74-81. https://doi.org/10.1038/nrneurol.2013.278 
Keith Conners C, Sitarenios G, Parker JDA, Epstein JN (1998) The revised Conners' Parent Rating Scale (CPRS-R): factor structure, reliability, and criterion validity. J Abnorm Child Psychol 26:257268. https://doi.org/10.1023/A:1022602400621

Lord C, Rutter M, Di Lavore P, et al (2012) Autism and Diagnostic Observation Schedule, Second Edition (ADOS-2) Manual (Part I): Modules 1-4

Loth E, Charman T, Mason L, Tillmann J, Jones EJH, Wooldridge C, Ahmad J, Auyeung B, Brogna C, Ambrosino S, Banaschewski T, Baron-Cohen S, Baumeister S, Beckmann C, Brammer M, Brandeis D, Bölte S, Bourgeron T, Bours C, de Bruijn Y, Chakrabarti B, Crawley D, Cornelissen I, Acqua FD', Dumas G, Durston S, Ecker C, Faulkner J, Frouin V, Garces P, Goyard D, Hayward H, Ham LM, Hipp J, Holt RJ, Johnson MH, Isaksson J, Kundu P, Lai MC, D'ardhuy XL, Lombardo MV, Lythgoe DJ, Mandl R, MeyerLindenberg A, Moessnang C, Mueller N, O’Dwyer L, Oldehinkel M, Oranje B, Pandina G, Persico AM, Ruigrok ANV, Ruggeri B, Sabet J, Sacco R, Cáceres ASJ, Simonoff E, Toro R, Tost H, Waldman J, Williams SCR, Zwiers MP, Spooren W, Murphy DGM, Buitelaar JK (2017) The EU-AIMS Longitudinal European Autism Project (LEAP): design and methodologies to identify and validate stratification biomarkers for autism spectrum disorders. Mol Autism 8:1-19. https://doi.org/10.1186/s13229-017-0146-8

Luiselli J, Happé F, Hurst H, et al (2013) Wechsler preschool and primary scale of intelligence. In: Encyclopedia of Autism Spectrum Disorders

Meiri G, Dinstein I, Michaelowski A, Flusser H, Ilan M, Faroy M, BarSinai A, Manelis L, Stolowicz D, Yosef LL, Davidovitch N, Golan H, Arbelle S, Menashe I (2017) Brief report: the Negev hospitaluniversity-based (HUB) autism database. J Autism Dev Disord 47: 2918-2926. https://doi.org/10.1007/s10803-017-3207-0

Muglia P, Filosi M, Da Ros L et al (2018) The Italian autism network (ITAN): a resource for molecular genetics and biomarker investigations. BMC Psychiatry 18:369. https://doi.org/10.1186/s12888-0181937-y

Mullen EM (1995) Mullen scales of early learning. AGS Edition: Manual and Item Administrative Books. Am Guid Serv Inc

Owens JA, Spirito A, McGuinn M (2000) The Children's Sleep Habits Questionnaire (CSHQ): psychometric properties of a survey instrument for school-aged children. Sleep 23:1043-1051. https:// doi.org/10.1111/j.1469-8749.2001.tb00204.x

Robins DL, Fein D, Barton ML, Green JA (2001) The modified checklist for autism in toddlers: an initial study investigating the early detection of autism and pervasive developmental disorders. J Autism Dev Disord 31:131-144. https://doi.org/10.1023/A:1010738829569

Sadiq S, Castellanos M, Moffitt J, et al (2019) Deep learning based multimedia data mining for autism spectrum disorder (ASD) diagnosis. In: 2019 International Conference on Data Mining Workshops (ICDMW). pp 847-854

Satterstrom FK, Kosmicki JA, Wang J et al (2020) Large-scale exome sequencing study implicates both developmental and functional changes in the neurobiology of autism. Cell 180:568-584.e23. https://doi.org/10.1016/j.cell.2019.12.036

Sparrow SS, Cicchetti DV, Balla DA (2005) Vineland adaptive behavior scales. 2nd

State MW, Levitt P (2011) The conundrums of understanding genetic risks for autism spectrum disorders. Nat Neurosci 14:1499-1506. https://doi.org/10.1038/nn.2924

Viezel K, Zibulsky J, Dumont R, Willis JO (2014) Bayley scales of infant and toddler development, 3rd edn. Encyclopedia of Special Education

Volden J, Smith IM, Szatmari P, Bryson S, Fombonne E, Mirenda P, Roberts W, Vaillancourt T, Waddell C, Zwaigenbaum L, Georgiades S, Duku E, Thompson A (2011) Using the Preschool Language Scale-IV to characterize language in preschoolers with ASD. Am J Speech Lang Pathol 20:200-208. https://doi.org/10. 1044/1058-0360(2011/10-0035

Zwaigenbaum L, Bauman ML, Choueiri R, Kasari C, Carter A, Granpeesheh D, Mailloux Z, Smith Roley S, Wagner S, Fein D, Pierce K, Buie T, Davis PA, Newschaffer C, Robins D, Wetherby A, Stone WL, Yirmiya N, Estes A, Hansen RL, McPartland JC, Natowicz MR (2015) Early intervention for children with autism spectrum disorder under 3 years of age: recommendations for practice and research. Pediatrics 136:S60-S81. https://doi.org/10.1542/ peds.2014-3667Es

Publisher's Note Springer Nature remains neutral with regard to jurisdictional claims in published maps and institutional affiliations.

\section{Affiliations}

Ilan Dinstein ${ }^{1,2,3} \cdot$ Ayelet Arazi ${ }^{2,3} \cdot$ Hava M. Golan ${ }^{3,4}$. Judah Koller ${ }^{5} \cdot$ Evan Elliott $^{6} \cdot$ Illana Gozes $^{7} \cdot$ Cory Shulman $^{8}$.

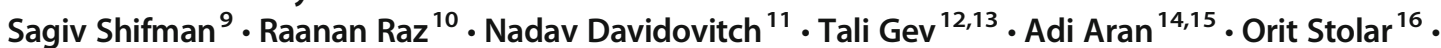
Esther Ben-Itzchak ${ }^{17}$. Irit Mor Snir ${ }^{13}$. Sandra Israel-Yaacov ${ }^{13,12} \cdot$ Nirit Bauminger-Zviely ${ }^{18}$ - Yoram S. Bonneh ${ }^{19}$. Eynat Gal $^{20}$ - Simone Shamay-Tsoory ${ }^{21}$ - Anat Zaidman Zait ${ }^{22,23}$ • Bat Sheva Hadad ${ }^{24,25} \cdot$ Raz Gross $^{26,27}$. Michal Faroy ${ }^{3,28}$ - Eitan Bachmat ${ }^{29}$ - Alal Eran ${ }^{30}$. Florina Uzefovsky ${ }^{1}$ - Hagit Flusser ${ }^{3,31}$ - Analya Michaelovski ${ }^{3,31}$. Stephen Z. Levine ${ }^{32}$. Arad Kodesh ${ }^{32,33}$. Doron Gothelf ${ }^{26,34,35}$. Daphna Marom ${ }^{26,36}$. Hagit Baris Feldman ${ }^{26,36}$. Dalit Ben Yosef ${ }^{37,38}$ - Aviva Mimouni Bloch ${ }^{26,39}$ - Yair Sadaka ${ }^{40}$. Chen Schtaierman ${ }^{41}$ - Michael Davidovitch ${ }^{42}$. Michal Begin ${ }^{43} \cdot$ Lidia V. Gabis $^{26,35,44}$ - Ditza Zachor ${ }^{16,26} \cdot$ Idan Menashe ${ }^{3,45} \cdot$ Ofer Golan $^{13,12,46}$ - Gal Meiri ${ }^{3,28}$

1 Department of Psychology, Ben Gurion University of the Negev, Beer Sheva, Israel

2 Department of Brain \& Cognitive Sciences, Ben Gurion University of the Negev, Beer Sheva, Israel

3 National Autism Research Center of Israel, Ben Gurion University of the Negev, Beer Sheva, Israel
4 Department of Physiology and Cell Biology, Faculty of Health Sciences, Ben Gurion University of the Negev, Beer Sheva, Israel

5 Seymour Fox School of Education, The Hebrew University of Jerusalem, Jerusalem, Israel

6 Molecular and Behavioral Neurosciences Lab, Faculty of Medicine, Bar-Ilan University, Safed, Israel 
7 The Elton Laboratory for Molecular Neuroendocrinology, Department of Human Molecular Genetics and Biochemistry, Sackler Faculty of Medicine, The Adams Super Center for Brain Studies and Sagol School of Neuroscience, Tel Aviv University, Tel Aviv, Israel

8 The Paul Baerwald School of Social Work and Social Welfare, The Hebrew University of Jerusalem, Jerusalem, Israel

9 Department of Genetics, The Institute of Life Sciences, The Hebrew University of Jerusalem, Jerusalem, Israel

10 Braun School of Public Health and Community Medicine, The Hebrew University of Jerusalem, Jerusalem, Israel

11 Department of Health Systems Management, School of Public Health, Ben Gurion University of the Negev, Beer Sheva, Israel

12 Department of Psychology, Bar-Ilan University, Ramat Gan, Israel

13 Autism Treatment and Research Center - The Association for Children at Risk, Givat Shmuel, Israel

14 Neuropediatric Unit, Shaare Zedek Medical Center, Jerusalem, Israel

15 The Hebrew University of Jerusalem, Jerusalem, Israel

16 The ALUT Autism Center, Shamir (Assaf Harofeh) Medical Center, Be'er Ya'akov, Israel

17 Bruckner Center for Research in Autism, Department of Communication Disorders, Ariel University, Ariel, Israel

18 School of Education, Bar-Ilan University, Ramat Gan, Israel

19 School of Optometry and Vision Science, Faculty of Life Science, Bar-Ilan University, Ramat Gan, Israel

20 Department of Occupational Therapy, Faculty of Social Welfare and Health Sciences, University of Haifa, Haifa, Israel

21 Department of Psychology, University of Haifa, Haifa, Israel

22 School of Education, Tel Aviv University, Tel Aviv, Israel

23 School of Population and Public Health, University of British Columbia, Vancouver, Canada

24 Department of Special Education, University of Haifa, Haifa, Israel

25 Edmond J. Safra Brain Research Center, University of Haifa, Haifa, Israel

26 Sackler Faculty of Medicine, Tel Aviv University, Tel Aviv, Israel

27 Division of Psychiatry, Sheba Medical Center, Tel Hashomer, Israel
28 Pre-School Psychiatry Unit, Soroka University Medical Center, Beer Sheva, Israel

29 Department of Computer Science, Ben Gurion University of the Negev, Beer Sheva, Israel

30 Department of Life Sciences, Ben Gurion University of the Negev, Beer Sheva, Israel

31 Zusman Child Development Center, Soroka University Medical Center, Beer Sheva, Israel

32 Department of Community Mental Health, University of Haifa, Haifa, Israel

33 Meuhedet Health Services, Tel Aviv, Israel

34 The Child Psychiatry Division, Edmond and Lily Safra Children's Hospital, Sheba Medical Center, Tel Hashomer, Israel

35 Sagol School of Neuroscience, Tel Aviv University, Tel Aviv, Israel

36 The Genetics Institute, Tel Aviv Sourasky Medical Center, Tel Aviv, Israel

37 IVF Lab and Wolfe PGD Stem Cell Lab, Tel Aviv Sourasky Medical Center, Tel Aviv, Israel

38 Department of Cell and Developmental Biology, Tel Aviv University, Tel Aviv, Israel

39 The Pediatric Neurology and Developmental Unit, Loewenstein Rehabilitation Medical Center, Ra'anana, Israel

40 Neuro-Developmental Research Centre, Beer Sheva Mental Health Centre, Ministry of Health, Beer Sheva, Israel

41 Child Development Center, Maccabi Health Services, Beer Sheva, Israel

42 Child Development, Medical Division, Maccabi Healthcare Services, Tel Aviv, Israel

43 Child Development Center, Leumit Healthcare Services, Jerusalem, Israel

44 Child Development Center, Edmond and Lily Safra Children's Hospital, Sheba Medical Center, Tel Hashomer, Israel

45 Department of Public Health, Ben Gurion University of the Negev, Beer Sheva, Israel

46 Autism Research Centre, Department of Psychiatry, University of Cambridge, Cambridge, UK 